\title{
USO DEL ENFOQUE POR PROCESOS EN LA ACTIVIDAD INVESTIGATIVA
}

\section{USE OF THE APPROACH BY PROCESSES IN THE RESEARCH ACTIVITY}

\author{
Jorge Iván Pérez Rave ${ }^{1} \quad$ Jairo Antonio Ruíz Córdoba ${ }^{2} \quad$ Carlos Mario Parra Mesa $^{3}$ \\ Recibido 19 de febrero de 2007, aceptado 26 de septiembre de 2007 \\ Received: February 19, 2007 Accepted: September 26, 2007
}

\begin{abstract}
RESUMEN
La realización de una investigación no sólo comprende actividades propias del campo de conocimiento de donde proviene la idea, sino también labores de gestión que permitan llevar a cabo de manera efectiva todas las etapas investigativas. Estas labores, en la mayoría de los casos subvaloradas en el campo investigativo, pero bien apreciadas en el contexto empresarial, son determinantes en el éxito de un proyecto de investigación. El enfoque por procesos constituye una de las principales bases de las prácticas de gestión que caracterizan a exitosas organizaciones empresariales y, en esta oportunidad, se exhibe la experiencia de aplicarlo al trabajo de campo de la investigación realizada por un grupo de la Universidad de Antioquia (Colombia). Este artículo pretende motivar y potenciar el desarrollo y/o la adaptación de modelos y técnicas de gestión en el ámbito investigativo, que propicien un desarrollo más eficaz y eficiente de las labores de investigación. En esta experiencia se define la misión del trabajo de campo, se identifican los procesos clave, se describe y se documenta cada uno de ellos y se realiza sensibilización y capacitación del equipo investigador en tales temas.
\end{abstract}

Palabras clave: Enfoque por procesos, trabajo de campo, gestión de la investigación, procesos clave.

\begin{abstract}
Research requires management tasks that allow effective execution of all the research stages. In most cases, these activities, which are decisive for the success of the project, are undervalued in the research context, but well appreciated in the enterprise context. The approach by processes is one of the main foundations of successful enterprise management practices. In this opportunity, the use of the aforementioned approach in the fieldwork stage of the research performed by a group of the Universidad de Antioquia (Colombia) is shown. This article intends to motivate and promote the development and/or adaptation of management models and tools, which will lead to a better development of the research. The mission of the fieldwork stage is defined, and the key processes are identified, described, and documented. Additionally, the research team is brought into awareness and trained in these topics.
\end{abstract}

Keywords: Approach by processes, fieldwork stage, research management, key processes.

\section{INTRODUCCIÓN}

El contexto actual, signado por la liberación de la economía, el avance tecnológico y la responsabilidad social exige de la academia soluciones expertas que conduzcan al mejoramiento de las condiciones de vida de la sociedad. Tal exigencia hace de la investigación un medio de innovación colectiva que posibilita la contribución de la academia a $\tan$ fausto fin.
Las universidades en particular cuentan con diversos grupos comprometidos con la generación de proyectos de investigación básica y aplicada, sobre los que subyace como objetivo principal el mejoramiento de la calidad de vida de la sociedad. Este impacto subyacente hace imperante que dichos proyectos se desarrollen de acuerdo con lo previsto y, de la misma manera, arrojen los resultados deseados, lo cual se constituye en una tarea compleja, puesto que el entorno es dinámico y cambiante, y reclama

\footnotetext{
1 Grupos de investigación: Ciencia y Tecnología Biomédica. Gestión de la Calidad. Universidad de Antioquia. Calle 67 No $53 \cdot 108$. Bloque 18 , oficina 126. Bello-Antioquia, Colombia. E-mail: jorgeprave@yahoo.es

2 Grupos de investigación: Ciencia y Tecnología Biomédica. Gestión de la Calidad. Universidad de Antioquia. Calle 67 No 53·108, Bloque 18, oficina 126. Medellín, Colombia. E-mail: jruiz@udea.edu.co

3 Grupos de investigación: Ciencia y Tecnología Biomédica, Gestión de la Calidad. Universidad de Antioquia. Calle 67 Nº 53·108, Bloque 18, oficina 126. Medellín, Colombia. E-mail: cmparra@udea.edu.co
} 
no solo actividades propias del campo de conocimiento de donde proviene la idea de investigación, sino también labores y aptitudes que permitan gestionar con eficacia $\mathrm{y}$ eficiencia todas las fases investigativas.

Si bien las diversas fases que intervienen en una investigación son fundamentales y, por lo mismo, ameritan el planear, hacer, verificar y actuar, en el caso de las investigaciones donde se emplea la encuesta como principal método de captura de la información, el trabajo de campo se constituye en una actividad compleja, extensa y de suma relevancia para el éxito de la misma, puesto que en él se capturan los datos requeridos para emitir resultados y generar conclusiones sobre las características de interés de la unidad de estudio, de modo que se cumpla con los objetivos trazados. Además, dicha fase se ve afectada por factores incontrolables como la disponibilidad de la fuente de información, que en un momento dado pueden afectar de modo negativo el progreso de la misma.

Dada la significativa incidencia de esta fase en el éxito de una investigación, es menester generar estrategias que garanticen en lo mayor posible su desarrollo esperado. Con miras a lo anterior, gestionar el trabajo de campo bajo un enfoque orientado a procesos, constituye una excelente vía para comprender el funcionamiento de las actividades que lo conforman, de las relaciones entre ellas y, de la misma manera, concentrar los recursos y los esfuerzos del talento humano hacia la consecución eficiente de los objetivos trazados.

Si bien es cierto que la etapa de interés tiene una fecha de inicio y de finalización, lo cual no es característico de un proceso, también lo es que durante la vigencia de la misma es perfectamente válido gestionarla bajo un enfoque por procesos, puesto que durante dicho periodo de tiempo el trabajo de campo se compone de una serie de actividades interrelacionadas, que transforman elementos de entrada en resultados y en las que interviene talento humano, recursos tangibles e intangibles, lo que se constituye en la definición formal de un proceso.

En esta oportunidad se exhibe la aplicación del enfoque por procesos al trabajo de campo de la investigación "Gestión ambiental de la amalgama dental en el departamento de Antioquia", realizada por el grupo Ciencia y Tecnología Biomédica (CTB) de la Facultad de Ingeniería de la Universidad de Antioquia (Colombia) y por la empresa New Stetic S.A. Dicha investigación surge a raíz de la problemática ambiental y de salud que representa el uso inadecuado del mercurio en la profesión odontológica $[1,2]$.
Este artículo constituye una base para motivar y propiciar el desarrollo y/o la adaptación de modelos y técnicas de gestión en el ámbito investigativo, a fin de que las labores se desarrollen con mayor efectividad y, en consecuencia, se contribuya de mejor manera al progreso de la sociedad.

\section{CONCEPTOS BÁSICOS}

A continuación se presentan algunas definiciones que facilitan una mejor comprensión de los demás apartados del presente artículo.

Trabajo de campo: El trabajo de campo es la etapa investigativa en la que se recolecta, mediante diversas técnicas, datos sobre las variables de interés de la unidad de estudio [3, 4]. En la investigación "Gestión ambiental de la amalgama dental en el departamento de Antioquia", la recolección de información se realizó en consultorios odontológicos del Departamento, mediante: toma de muestras de cabello del personal, mediciones de vapor de mercurio, entrevistas y observación directa sobre características locativas de la entidad.

Enfoque orientado a procesos: El enfoque por procesos conlleva a concebir la organización, en este caso el trabajo de campo de una investigación, como un sistema compuesto por un conjunto de procesos interrelacionados que buscan un objetivo global que cumplir. Este enfoque consiste en identificar los procesos y sus interrelaciones, para girar el ciclo PHVA (Planear, Hacer, Verificar y Actuar) sobre ellos y mejorar su efectividad, a fin de satisfacer las necesidades de todas las partes interesadas [5]. Además, parte del principio de que los resultados anhelados se logran con mayor eficiencia cuando las actividades y los recursos se gestionan como procesos [6]. Entre los beneficios que trae consigo este esquema de gestión se encuentran: la lateralización de la organización $[7,8]$, la orientación al cliente, el trabajo en equipo y la mejora continua.

Proceso: Como proceso puede entenderse un conglomerado de actividades interrelacionadas, mediante las cuales se agrega valor a unas entradas (materiales o inmateriales), suministrando luego productos, servicios e información a un cliente externo o interno [9]. De acuerdo con Martín y Mallol [10], un proceso es un conjunto de recursos (personal, finanzas, instalaciones, equipos, técnicas, métodos) y actividades relacionadas entre sí, que transforman elementos de entrada en elementos de salida [10]. Andriani [11] se refiere a proceso como un conglomerado de operaciones, conformado por materiales, 
maquinaria, métodos y personas que, agregando valor, buscan transformar la materia prima en producto terminado útil para el consumidor [11].

Clasificación de los procesos: Existen diversas categorías de procesos, pero generalmente se clasifican en: estratégicos, clave y de apoyo [12].

Los procesos clave, también conocidos como procesos primarios, críticos o misionales, son aquellos que inciden directamente en la satisfacción de los clientes y están estrechamente asociados a la razón de ser de la organización. En el ámbito del trabajo de campo, estos procesos pueden interpretarse como aquellos directamente relacionados con la actividad investigativa.

Los procesos de apoyo son los encargados de apoyar y respaldar a los procesos clave, de modo que éstos puedan cumplir con la misión que los caracteriza. Haciendo énfasis en el trabajo de campo, la gestión de recursos, de contratos y de viajes, corresponden a procesos de apoyo, pues no aportan valor, sino que son necesarios para el buen funcionamiento de los procesos clave.

La categoría de procesos estratégicos hace referencia a aquellos encargados del pilotaje de la organización, éstos permiten definir la estrategia, los objetivos y las políticas, y desplegarlas a los diferentes niveles de la organización.

En el contexto de interés, el investigador principal puede verse como el encargado del pilotaje de la investigación $\mathrm{y}$, por tanto, llevará consigo el compromiso de la alta dirección. Además, le corresponderá ejercer un liderazgo activo que posibilite el trabajo en equipo y el establecimiento $\mathrm{y}$ despliegue de las directrices a seguir.

\section{METODOLOGÍA}

Definición de la misión del trabajo de campo: Con base en los objetivos y en la metodología del proyecto "Gestión ambiental de la amalgama dental en el departamento de Antioquia", y dando respuesta a cinco interrogantes fundamentales, se definió la misión del trabajo de campo. Las preguntas empleadas para tal fin fueron las siguientes: ¿qué se realizará?, ¿cómo se hará?, ¿dónde se llevará a cabo?, ¿para quién se realizará? y ¿a quién se le realizará?

Modelo de procesos del trabajo de campo: Partiendo de la misión del trabajo de campo se listaron las actividades que intervienen en su desarrollo. Luego, con base en la secuencia, la relevancia, la repetitividad, los recursos asociados a dichas actividades y el talento humano implicado, se analizaron y se agruparon en diversos procesos.

Es de resaltar que si bien existen tres tipos de procesos: clave, de apoyo y estratégicos, el alcance del presente trabajo constituye una exploración inicial que se delimitó a los procesos clave, pues en ellos se desarrolla propiamente la actividad investigativa y, por lo mismo, son los directamente asociados a la misión del trabajo de campo; contrario a los procesos estratégicos y de apoyo, pues los primeros se traducen en el pilotaje de la investigación y, los segundos, abordan básicamente la gestión de recursos, de viajes y de contratos del personal, para lo cual el centro encargado en la Facultad de Ingeniería de la Universidad de Antioquia (Centro de Investigaciones Ambientales y de Ingeniería) tiene debidamente establecidos los procedimientos.

Descripción de los procesos: Inicialmente se definió la misión de los procesos identificados en la etapa anterior $y$, a partir de ello, se identificaron las entradas, las salidas y los recursos asociados a cada uno de ellos. Finalmente, se asignó el responsable de cada proceso.

Documentación de los procesos: Con el propósito de facilitar la comprensión del modelo de procesos del trabajo de campo y la capacitación del equipo investigador en tal tema, se consideró necesario documentar los procesos. Para ello, se empleó la técnica del diagrama de flujo, la cual, de manera sencilla, permite representar gráficamente la secuencia de las actividades que conforman cada proceso [13].

Sensibilización y capacitación del equipo investigador: En esta fase se dictó una charla de sensibilización acerca de la importancia de gestionar el trabajo de campo desde un enfoque por procesos $y$, posteriormente, se realizaron dos actividades dedicadas a la capacitación y al entrenamiento del equipo investigador en el modelo desarrollado.

Validación del modelo de procesos y elaboración de la versión final: Puesto que la investigación para la cual se desarrolló el presente trabajo comprendió la elaboración de cuestionarios estructurados para colectar datos sobre la gestión de la amalgama dental en el departamento de Antioquia, los cuales fueron validados mediante una prueba piloto en seis consultorios odontológicos, se aprovechó también dicha actividad para validar el modelo elaborado. Finalizada la prueba piloto, el equipo investigador analizó el desarrollo del trabajo de campo bajo este enfoque, generó sugerencias de mejoramiento y, luego de los ajustes, elaboró la versión final bajo la cual se abordó el trabajo de campo de la investigación. 


\section{RESULTADOS}

Misión del trabajo de campo: A continuación se exhibe la razón de ser del trabajo de campo del proyecto "Gestión ambiental de la amalgama dental en el departamento de Antioquia". En ella se distingue claramente la respuesta a los interrogantes planteados en la primera fase metodológica.

Misión: "Capturar datos sobre la gestión de la amalgama dental en el departamento de Antioquia, que permitan emitir resultados y generar conclusiones para lograr los objetivos trazados por el equipo investigador. Lo anterior, mediante entrevistas al odontólogo responsable de la entidad odontológica y/o al auxiliar más relacionado con el manejo de la amalgama dental, así como también a través de la observación y el registro de las condiciones locativas de los consultorios odontológicos. Todo ello, garantizando el profesionalismo en las labores realizadas".

Modelo de procesos del trabajo de campo: En la figura 1 se ilustra el modelo de procesos clave del trabajo de campo, el cual está conformado por: solicitud de citas, asignación y control de citas, realización de encuestas y toma de muestras y almacenaje de resultados y envío de muestras al laboratorio.

\section{Descripción de los procesos}

A continuación se describe cada uno de los procesos clave que conformaron el trabajo de campo de la investigación.

Proceso de solicitud de citas: Este proceso tiene como misión comunicar vía telefónica a la unidad de información sobre el proyecto y solicitar una disposición favorable por parte de los servicios odontológicos, que permita la realización de encuestas y la toma de muestras ambientales $\mathrm{y}$ biológicas en sus instalaciones.

De la figura 2 se observa que la entrada de este proceso es un formato en blanco (ver tabla 1) previamente diseñado en la investigación para registrar las citas acordadas con el representante de cada entidad odontológica. Una vez que ingresa dicho formato al proceso de solicitud de citas, se desarrolla una serie de actividades de comunicación con la entidad odontológica y, finalmente, como salida del proceso, se tiene el mismo formato debidamente diligenciado.
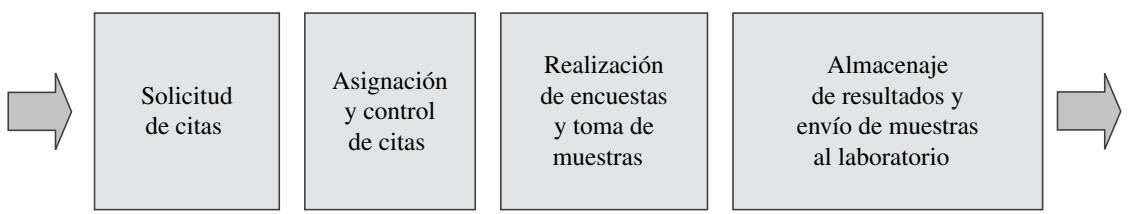

Figura 1. Procesos clave del trabajo de campo de la investigación.
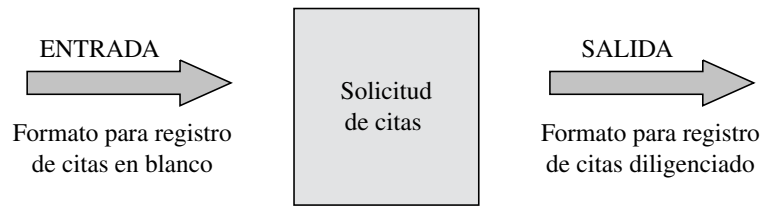

Figura 2. Proceso de solicitud de citas.

Tabla 1. Formulario de solicitud de citas

\begin{tabular}{|l|c|c|c|c|c|c|c|c|c|}
\hline \multicolumn{7}{|c|}{ FORMULARIO PARA SOLICITUD Y REGISTRO DE CITAS } \\
DILIGENCIADO POR: \\
\hline $\mathbf{N}^{\mathbf{0}}$ & Día & Mecha de cita & Año & Hora & Entidad & Municipio & Dirección & Teléfono & Representante \\
\hline & & & & & & & & & \\
\hline & & & & & & & & & \\
\hline & & & & & & & & & \\
\hline
\end{tabular}


Proceso de asignación y control de citas: En la figura 3 puede verse la modelación del proceso de asignación y control de citas, el cual parte del formato de solicitud de citas debidamente diligenciado. Luego, mediante una aplicación básica en Excel, se procesa la información recolectada, asignando a cada entidad un código que permite identificar información general sobre el consultorio odontológico (depositada en el formulario de solicitud de citas) y el estado del mismo respecto a la gestión de la amalgama dental.

Además de lo anterior, en este proceso también se preparan los materiales necesarios para efectuar el trabajo de campo. Como resultado se despacha al encuestador hacia la unidad odontológica, completamente preparado para realizar la labor de campo.

Proceso de realización de encuestas y toma de muestras: En la figura 4 se presenta la modelación básica de este proceso, cuya entrada corresponde al encuestador, ubicado en la entidad odontológica y debidamente preparado para realizar el trabajo de campo.

El presente proceso se desarrolla básicamente en la entidad odontológica objeto de estudio, el encuestador realiza una serie de actividades investigativas $\mathrm{y}$, como resultado del proceso, se tienen datos sobre la gestión de la amalgama dental en el consultorio odontológico, registrados en cuestionarios y en muestras biológicas y ambientales.

Proceso de almacenaje de resultados y envío de muestras al laboratorio: Una vez que el encuestador aplica los cuestionarios y toma las muestras ambientales y biológicas, regresa a la Universidad y, seguido, el responsable del presente proceso efectúa control de calidad a las encuestas y a las muestras. Como resultado se tienen los cuestionarios almacenados en un lugar seguro y las muestras ambientales y biológicas son enviadas a los laboratorios para que sean analizadas. En la figura $5 \mathrm{se}$ ilustra el proceso de almacenaje de resultados y envío de muestras al laboratorio.
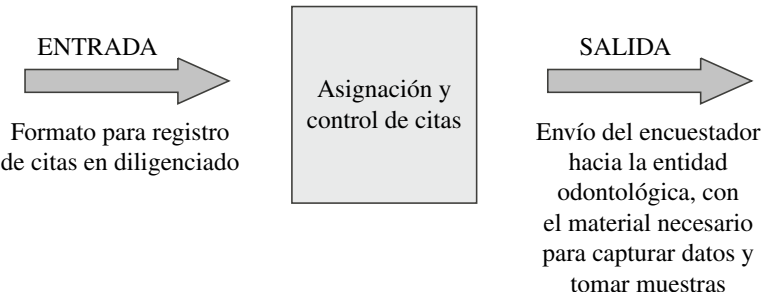

Figura 3. Proceso de asignación y control de citas.
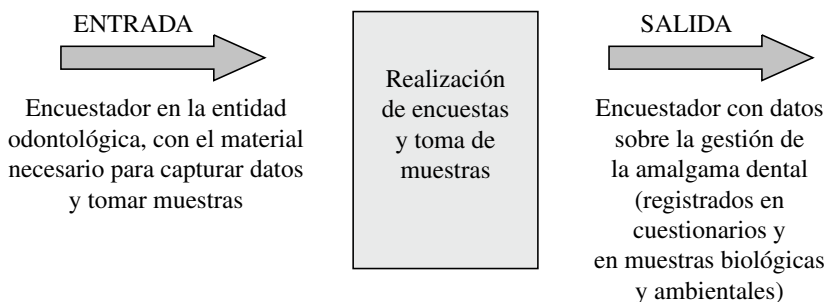

Figura 4. Proceso de realización de encuestas y toma de muestras. Fuente: elaboración propia.
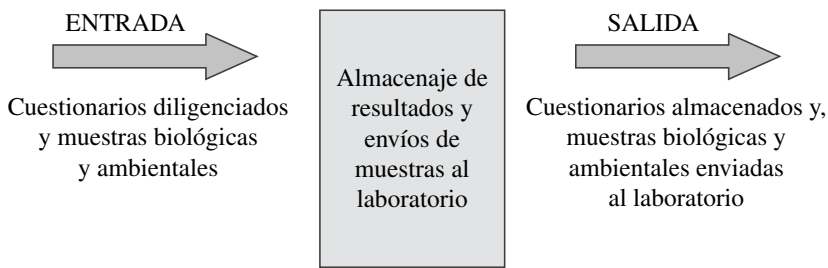

Figura 5. Proceso de almacenaje de resultados y envío de muestras al laboratorio. Fuente: elaboración propia. 


\section{Documentación de los procesos}

A continuación se muestra el diagrama de flujo de los 4 procesos clave del trabajo de campo y se comentan las actividades que los conforman:

Solicitud de citas: En este proceso el investigador responsable se contacta con la entidad odontológica, comenta sobre el proyecto, solicita autorización para realizar la encuesta y la toma de muestras y acuerda la fecha de la cita. Estas actividades se repiten hasta completar entre 10 y 15 citas, a fin de realizar una programación más eficiente, pues en algunos casos, debido a la falta de disponibilidad de la unidad de información, era preciso modificar la fecha inicialmente acordada para la cita.

En otras palabras, puede decirse que cada 10 o 15 citas acordadas, el proceso arroja un producto: formulario (tabla 1) diligenciado. Además, teniendo en cuenta que el equipo investigador debía ajustarse a dicha disponibilidad y con el ánimo de que tal situación no afectara drásticamente el progreso del trabajo de campo, se daba libertad a la fuente de información para que seleccionara la fecha y hora de la cita en un lapso de tiempo determinado. En la figura 6 se exhibe el diagrama de flujo del presente proceso.

Asignación y control de citas: En la figura 7 se exhibe el diagrama de flujo del presente proceso, que parte de la información recopilada en el formulario de solicitud de citas, la cual se archiva en el computador a través de una aplicación básica denominada "asignación y control de citas". Luego se asigna el respectivo encuestador y media hora antes del momento en que debe desplazarse hacia la entidad se realiza una reunión con el director de la investigación para entregar el material de trabajo, verificar detalles y autorizar el desplazamiento del encuestador hacia el consultorio odontológico.

Es de resaltar que dentro del material de trabajo del encuestador, conformado por cuestionarios, lapicero, maletín, sobres de manila, bomba para medición de vapores de mercurio, cartas de presentación, entre otros, también se encuentra una credencial (ver tabla 2) que proporciona la información requerida para viajar hacia el consultorio odontológico y para diligenciar información general sobre la encuesta (código de la encuesta, encuestador, municipio, etc.).

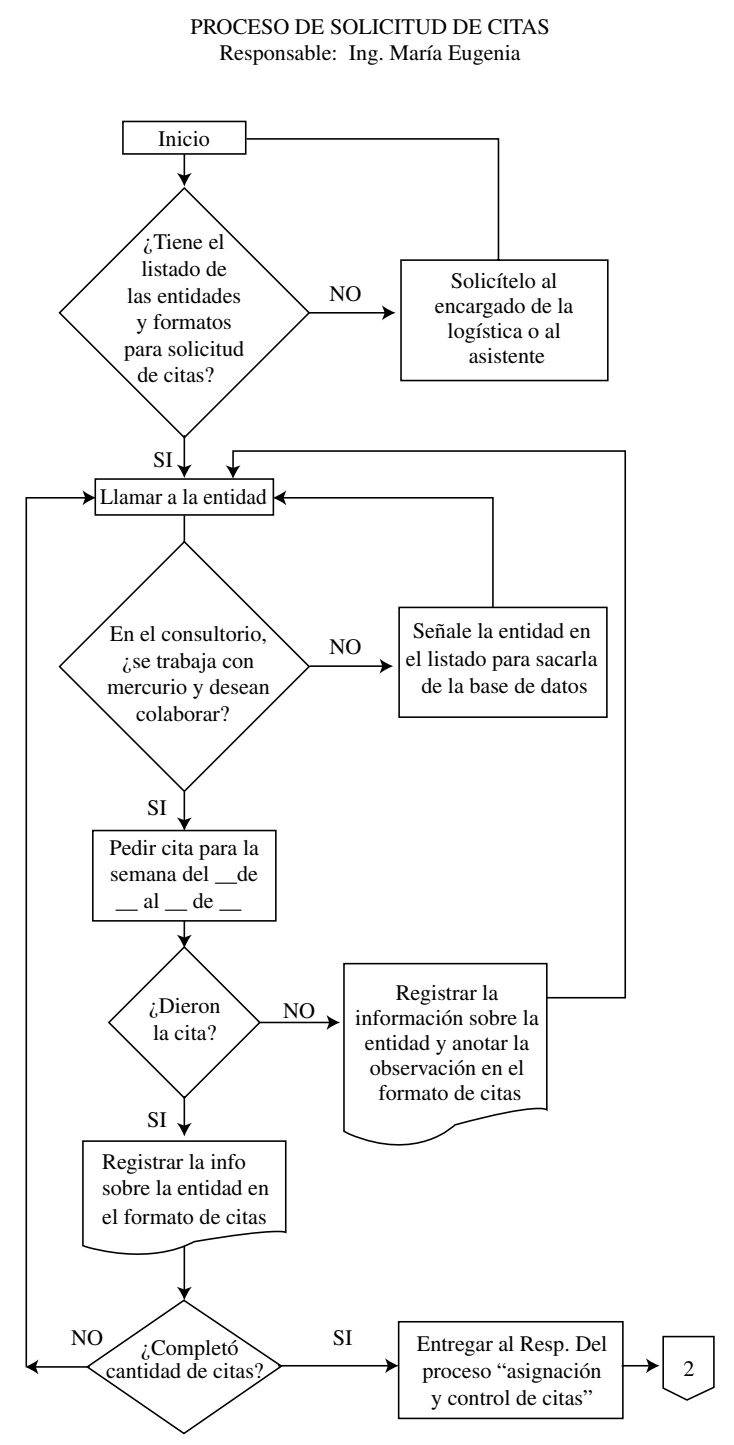

Figura 6. Diagrama de flujo del proceso de solicitud de citas. Fuente: elaboración propia.

Tabla 2. Credencial de la cita.

\begin{tabular}{|l|l|l|l|l|l|l|l|l|l|l|}
\hline \multicolumn{1}{|c|}{ CREDENCIAL DE LA CITA } \\
\hline Código & Día & Mes & Año & Hora & Encuestador & Municipio & Entidad & Dirección & Teléfono & Representante \\
\hline & & & & & & & & & & \\
\hline
\end{tabular}


PROCESO DE ASIGNACIÓN Y CONTROL DE CITAS

Responsable: Est. Jorge P. Rave

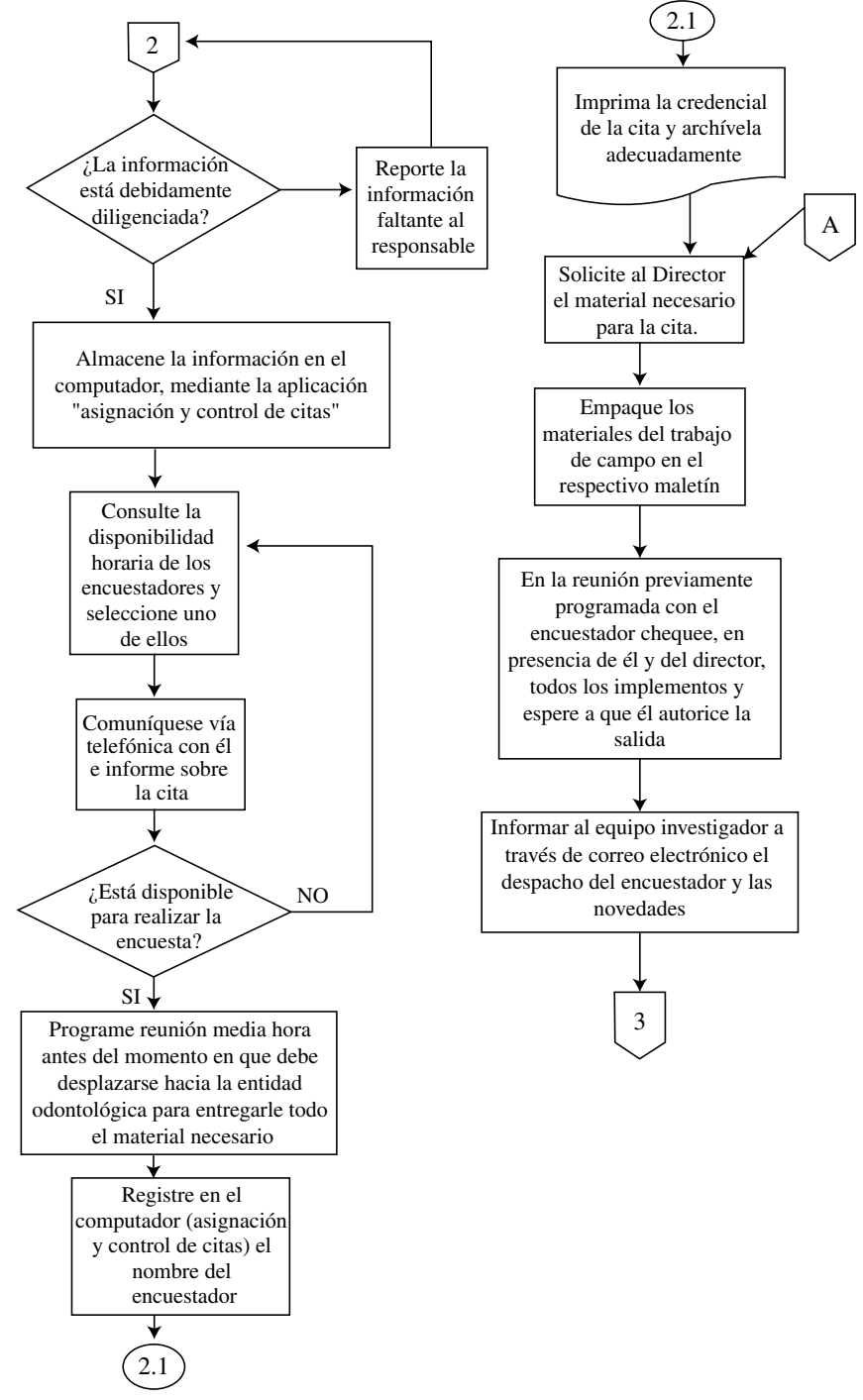

Figura 7. Diagrama de flujo del proceso de asignación y control de citas. Fuente: elaboración propia.

Realización de encuestas y toma de muestras: La figura 8 exhibe las actividades a desarrollar en este proceso, así como la secuencia entre ellas. En él, mediante dos cuestionarios estructurados, elaborados en una etapa anterior al trabajo de campo, se desarrolla propiamente la captura de los datos requeridos por el equipo investigador. A través de uno de ellos se colectan datos proporcionados por el odontólogo responsable de la entidad y/o el auxiliar que mayor contacto tiene con la amalgama dental; mediante el otro cuestionario se registran datos, resultado de la observación de las condiciones locativas de la entidad.

Igualmente, en este proceso se colectan otros datos sobre la entidad, a partir de la toma de muestras de cabello al personal del consultorio odontológico y de la medición de vapores de mercurio en el sitio.

Con el ánimo de garantizar la eficacia en la recolección de los datos, se elaboraron instructivos, tanto para la aplicación de los cuestionarios como para la toma de muestras ambientales y biológicas. 


\section{PROCESO DE REALIZACIÓN DE ENCUESTAS Y TOMA DE MUESTRAS Responsable: Encuestador}

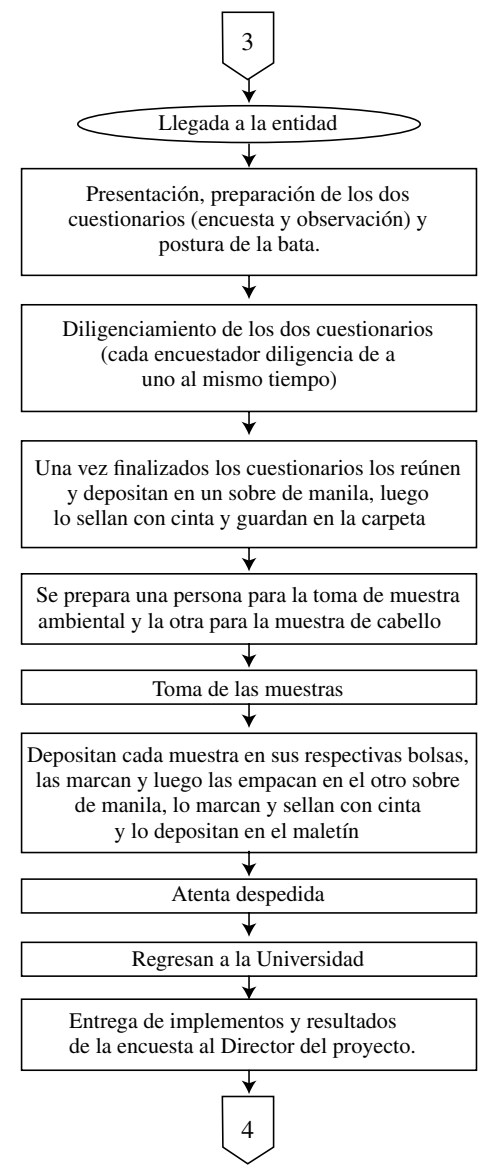

Figura 8. Diagrama de flujo del proceso de realización de encuestas y toma de muestras. Fuente: elaboración propia.

\begin{abstract}
Almacenaje de resultados y envío de muestras al laboratorio: De la figura 9 se observa que una vez que el encuestador regresa de efectuar el trabajo de campo, el responsable del presente proceso realiza control de calidad a las encuestas y a las muestras. En caso de que exista información faltante, se consulta con el encuestador y, de ser necesario, se llama o se viaja nuevamente a la entidad para colectar lo faltante. Luego de lo anterior, se procede a almacenar en un sitio seguro los cuestionarios debidamente diligenciados; además, se verifica si hay almacenadas como mínimo 30 muestras y, en caso afirmativo, se envían al respectivo laboratorio. Se optó por enviar grupos de muestras ambientales y biológicas a los laboratorios, en lugar de una por una, por petición de los laboratorios y con el fin de racionalizar viajes, costos y tiempo.
\end{abstract}

PROCESO DE ALMACENAJE DE RESULTADOS Y ENVÍO DE MUESTRAS AL LABORATORIO

Responsable: Prof. Jairo Ruíz Córdoba

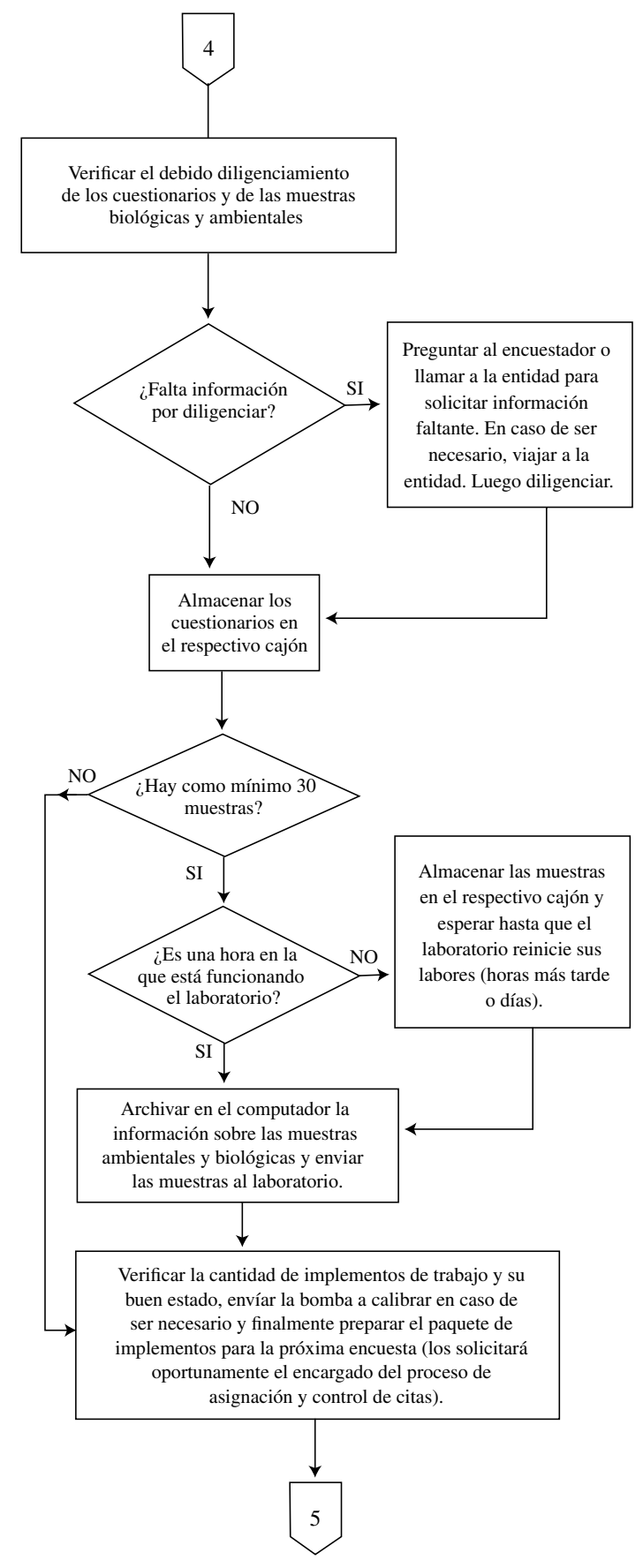

Figura 9. Diagrama de flujo del proceso de almacenaje de resultados y envío de muestras al laboratorio. Fuente: elaboración propia. 


\section{CONCLUSIONES}

- Con la aplicación del enfoque por procesos al trabajo de campo de la investigación "Gestión ambiental de la amalgama dental en el departamento de Antioquia", disminuyó el enfoque reactivo que caracterizó al equipo investigador en trabajos anteriores, se facilitó la comunicación entre las personas y se logró una mejor integración de las fortalezas del talento humano. Cada persona fue consciente del lugar que ocupó dentro del trabajo de campo, de la relación cliente-proveedor, de las responsabilidades que le fueron asignadas $y$, asimismo, cada investigador tuvo mayor autonomía tanto para la realización de sus tareas como para el mejoramiento de las mismas. Todo lo anterior, comparado con experiencias pasadas que carecieron de la aplicación de este enfoque, se vio reflejado en un equipo investigador más motivado, en la disminución del tiempo para realizar las tareas, en la minimización de defectos como: incumplimiento de la citas, impuntualidad, falta de disponibilidad del material de trabajo, y en una mayor satisfacción tanto de la unidad de información (odontólogos y/o auxiliares) como del grupo de investigación.

- El enfoque por procesos es una herramienta efectiva en el ámbito empresarial y su uso puede replicarse hacia el contexto investigativo, con el propósito de potenciar el trabajo en equipo, tener una mejor comprensión de las actividades investigativas y contribuir a una gestión más eficaz y eficiente de las mismas, de modo que se logren los objetivos trazados en el momento oportuno, se racionalicen los recursos y se responda satisfactoriamente a los requerimientos de los diferentes actores implicados. No obstante, al igual que en el ámbito empresarial, si la implementación del enfoque de interés no cuenta con el compromiso de la alta dirección, reflejada en este caso en el investigador principal, todos los esfuerzos que se emprendan tenderán al fracaso.

- Si bien la aplicación del enfoque por procesos en las labores investigativas aún se encuentra en su infancia, con este trabajo se abren diversas posibilidades de estudio que aborden desde esta perspectiva otras fases investigativas, como, por ejemplo, la revisión literaria. Igualmente, la experiencia presentada en este artículo puede ser replicada o adaptada fácilmente al trabajo de campo de otros proyectos de investigación, a fin de posibilitar una gestión más efectiva.

\section{REFERENCIAS}

[1] S.C. Bello, M.C. Rodríguez, D.R. Fernández, A. del C. Vásquez, A.M. Ocando, J.R. Contreras y V.A. Granadillo. "Niveles de mercurio en cabello de individuos expuestos ocupacionalmente en el área odontológica". Acta odontológica. Vol. 40 $\mathrm{N}^{\mathrm{o}}$ 2, pp. 123-128. Venezuela. 2002.

[2] I. Morales Fuentes y R. Reyes Gil. "Mercurio y salud en la odontología”. Rev. Saúde Pública. Vol. 37 No 2, pp. 266-272. São Paulo, Brasil. 2003.

[3] B. Hernández y H. Velasco. "Encuestas transversales". Salud pública. Vol. 42 N 5, pp. 447-455. México. Septiembre-octubre, 2000.

[4] M.J. González Rivas. "El uso social del juguete en Ciudad Rodrigo: una aproximación etnográfica en la posguerra española”. Aula: Revista de enseñanza e investigación educativa. Vol. 13, pp. 141-163. 2001.

[5] J.I. Pérez Rave. "Aproximación del enfoque por procesos al contexto universitario I". Entornoempresarial. Venezuela. Vol. 5. Julio 2006. URLs: http://www.entorno-empresarial.com

[6] Icontec. Instituto Colombiano de Normas Técnicas y Certificación. Sistemas de Gestión de la Calidad. Fundamento y vocabulario. ISO 9000:2000. 2 de diciembre, 2000.

[7] J.R. Galbraith. "The reconfigurable organization". The Organization of the Future, The Drucker Foundation, pp. 87-98. Jossey-Bass Publishers, San Francisco, EUA. 1997.

[8] F. Hesselbeim. "The circular organization". The Organization of the Future, the Drucker Foundation, pp. 81-86. Jossey-Bass Publishers. San Francisco, EUA. 1997.

[9] R. Cooper. "When Lean Enterprises Collide: Competing through Confrontation". Harvard Business School Press. 1995.

[10] S. Martín y A. Mallol. "Creación de un agente para la gestión de procesos". IX Congreso Nacional de Informática Médica. Informe, pp. 249. 2002.

[11] C. Andriani, R. Biasca y M. Rodríguez. "Nuevo sistema de gestión para lograr Pymes de clase 
mundial". México. Editorial. Norma, pp. 62. 2003.

[12] L. Dueñas, H. García y J. Espinoza. "Caracterización de un Sistema de Gestión de Información Científico Tecnológica con enfoque a procesos: garantía para la mejora continua. Estudio de caso". Congreso Internacional de Información. Palacio de las Convenciones Ciudad de La Habana, Cuba. 12 al 16 de abril, 2004.

[13] P. A therton. Manual para sistemas y servicios de información. París: Unesco. 1978. 